
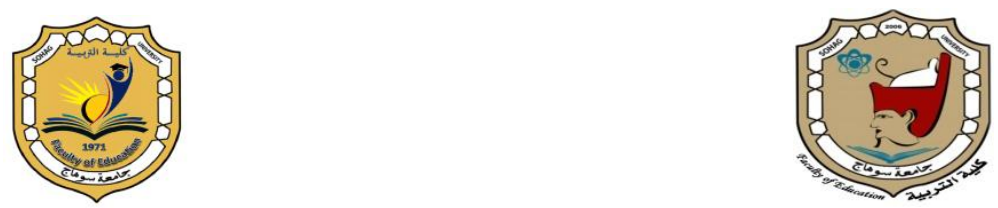

كلية التربية

جامعة سوهاج

المجلة التريوية

\title{
في أهمية تعليم التفلسف
}

\section{إعداد}

أ.د / محمد سعيد أحمد أحمد زيلدان

أستاذ المناهج وطرق تلريس القلسفة

كلية التربية - جامعة حلوان

تاريخ القبول : 10 أغسطس اYY Y

تاريخ الاستلام : r أغسطس ابr Tr

DOI: 10.12816/EDUSOHAG.2021. 
جامعة الحياة.

In Importance of Teaching Philosophize

Dr. Mohammed saed Ahmed Ahmed Zedan

Professor of Curricula and Philosophy Teaching methods,

Faculty of Education Helwan University.

\section{Summary:}

Teaching Philosophize to high School Students is a necessity; preparing them For entry of the University or life University. 
إن الحياة غذاء الفلسفة اليومي، والفلسفة غذاء الحياة الدائم، ثمة علاقات عضوية متثابكة، لا يستطيع طرف أن ينفصل عن الآخر، حتى بإرادته الحرة.. قانون الحاجة

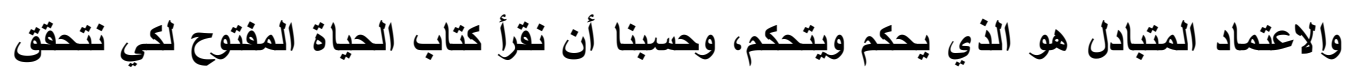

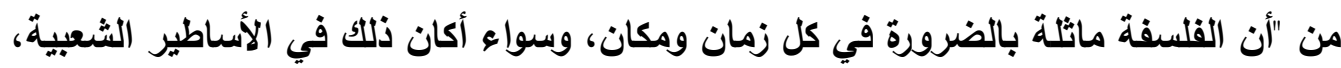

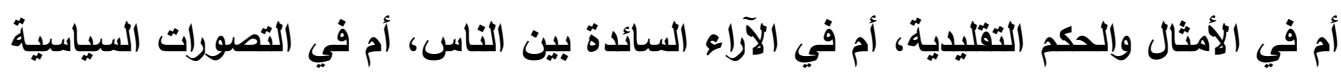

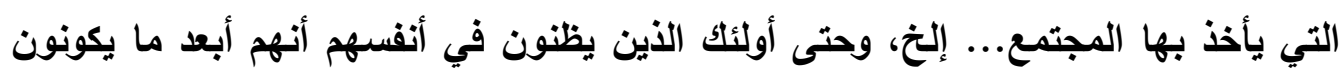

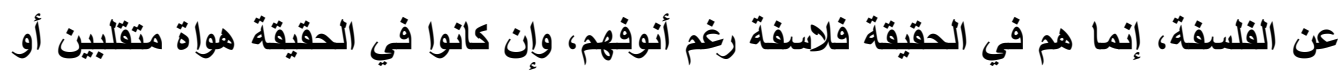
مفكرين مذبذبين ينتقلون من فكرة إلى أخرى، دون أن يكون لايهم شعور واضح بما يفعلون!

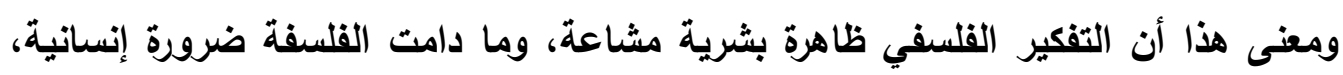
فهيهات لأحد أن يستغنى عنها أو أن يتخلص منها"(1)؛ لأنها آية الحكمة الإنسانية.

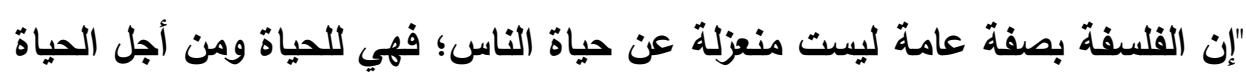

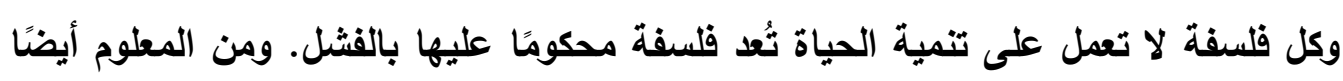

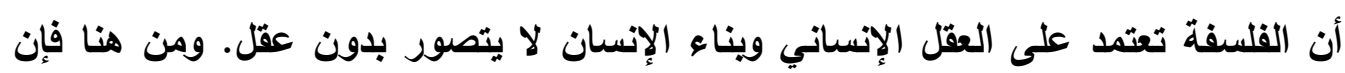

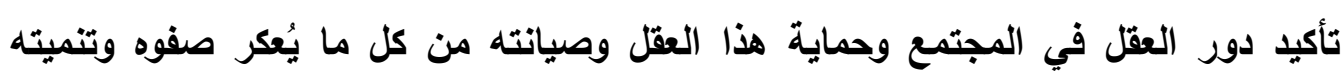

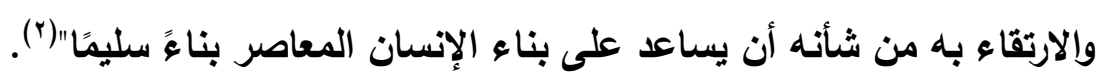

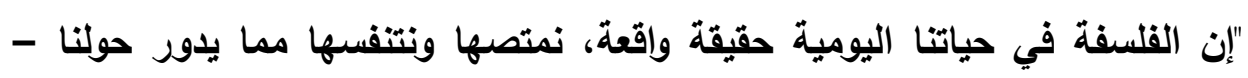

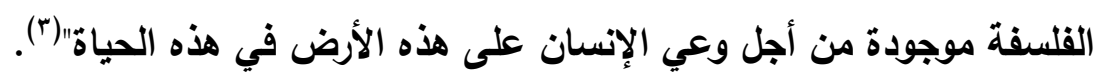

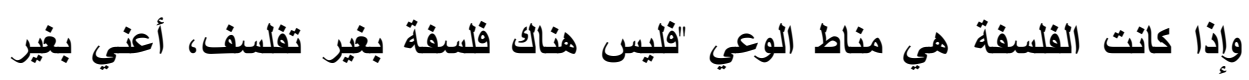
مشاركة في مسائلها، وتجربة حية لمشكلاتها. صحيح أن هناك عددًا لا حصر له من الحقائق

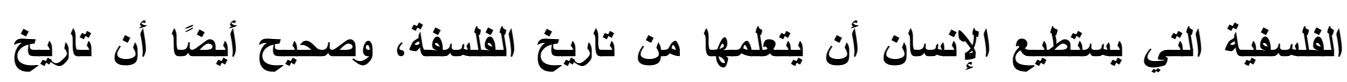

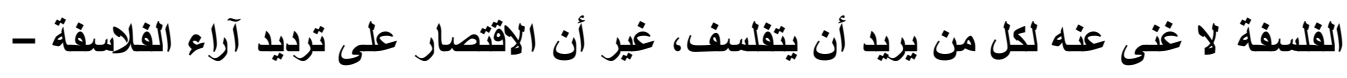

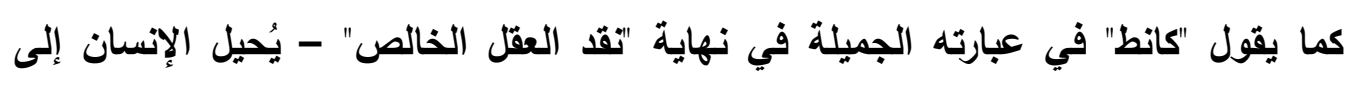

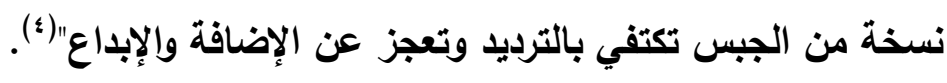

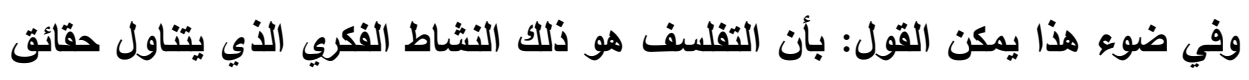
الحياة باستخدام النظرة النقدية القاحصة بدلاً من قبول الأمور على علاتها. 
والواقع أن التفكير الفلسفي حكمة في التفكير، ويقين في البحث، وضرورة إنسانية

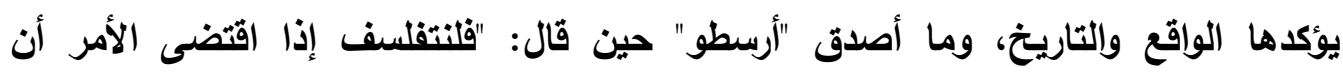

نتفلسف فإذا لم يقتض الأمر التفلسف وجب أن نتفلسف لنتبت أن التفلسف لا ضرورة لهة|(ه). ولعل من الخير أن نقر منذ الباية بأهمية التفلسف وضرورة التفكير الفلسفي. وفي ضوء هذا يُقرر "ديكارت"(") "أن الفلسفة وحدها هي التي تميزنا عن الأقوام المتوحشين

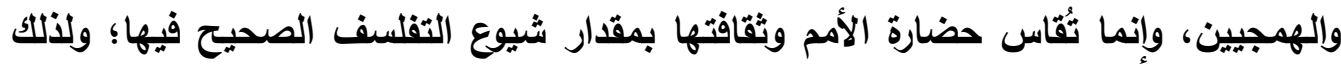

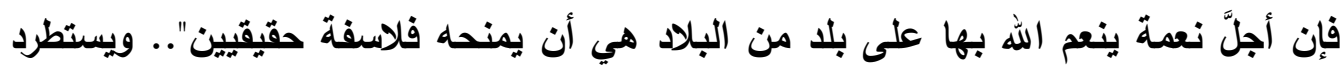

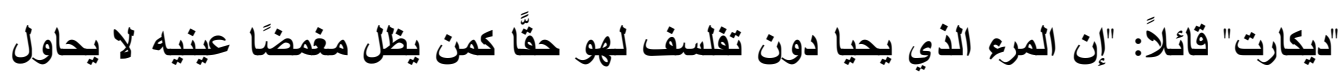

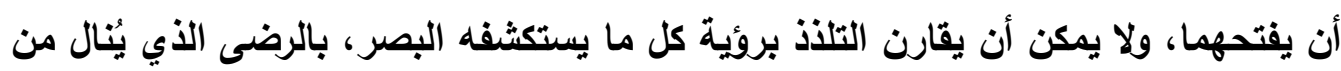

معرفة الأثشياء التي تتكثف لنا بالفلسفة".

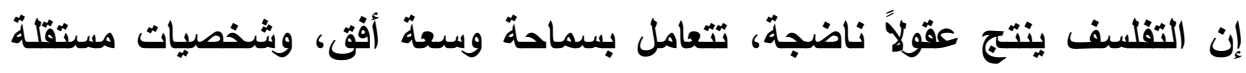

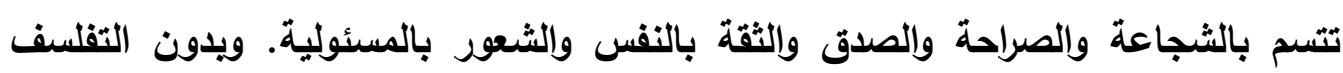

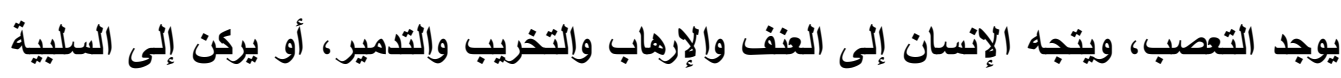

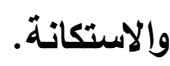
نحن أحوج ما نكون إلى التفلسف؛ لأنه يصنع التقام. إن التفلسف هو السبيل السريع

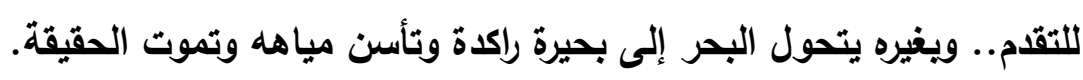

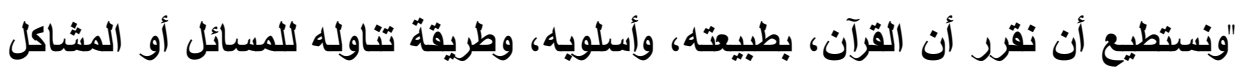

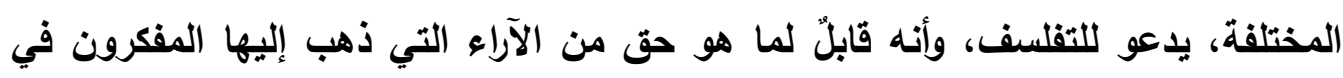

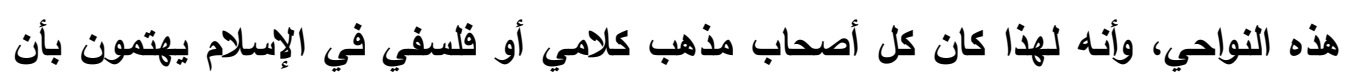

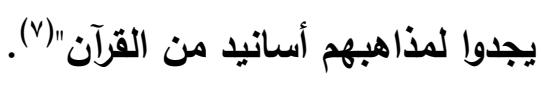
وكان الرسول (صلى الله عليه وسلم) يشجع أصحابه على التفكير والاستدلال العقلي

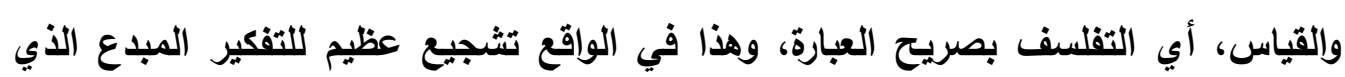

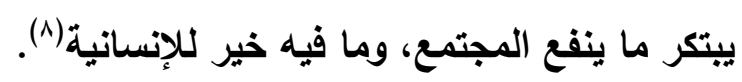

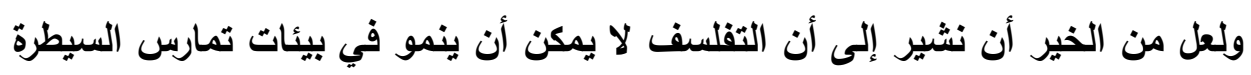

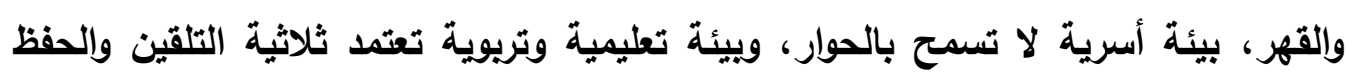

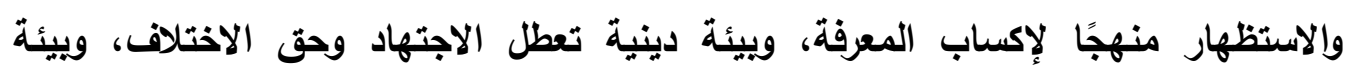


سياسية تعطل حرية التفكير والتعبير، وأجهزة إعلام لا تؤمن بتتوع الأفكار والآراء وتعدد أساليب الوصول إلى الحقيقة.

ويؤكد "كانط" إن مهمة معلم القلسفة لا تتحصر في تعليم تلاميذه بعض الأفكار

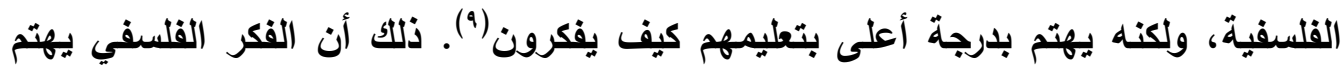
بالسؤال كيف نفكر لا فقط بماذا نفكر؟ وفي ضوء هذا يقرر "توملين Tomlin"(·)": لن تتعلم مني الفلسفة، ولكن القضية كيف تتفلسف؟ فالفلسفة ليست أفكارًا ألقتها، أو أكررها عليك، بل كيف تفكر؟ فلتفكروا لأنفسكم، ولتبحثُوا عن الحقيقة، ولتعتمدوا على أنفسكم". إن تعليم التقلسف يحقق هدفًا تريويًا محدًا حيث يدرك الطلاب أن الحقيقة أكبر من أن يحيط بها عقل وإح، حتى ولو كان عقلاً عبقريًا كعقل "أرسطو" أو "ديكارت" أو "كانط" أو "هيجل"، ومن سقطات العقول الكبيرة يكتسبون فضيلتي التواضع والتسامح الفكري، فينأون بأنفسهم عن التعصب الأعمى لمبدأ أو فكرة، ويندفعون إلى التعامل مع الفكر بعقل ديالكتيكي نقدي.

إن التقلسف قبل كل شيء أداة تسمح للطالب بالتفكير النقدي، واستقلالية الفكر لايه، وتفتيح آفاق المعرفة وسبل الحصول عليها، ومواجهة الفكر بالفكر. فالتفلسف يدعوه إلى بـ التفكير وممارسة الملكة التحليلية والنقدية بلدل سرد المعلومات التي تلقاها في الاروس. على ضوء هذا، قد يكتسب تدريس القلسفة بالمرحلة الثانوية أهمية جديدة، ولا يعود مجرد سرد للأفكار والنظريات الفلسفية، بل قد يُصبح التمرس والتدريب على مهارات التفكير الفلسفي - على الأقل - مفتاحًا لتلك العملية التي يحقى بها الإنسان انتقاله من المملكة الحيوانية إلى المملكة الإنسانية. إنتي أعتقد أن تعليم التفلسف لطلاب المرحلة الثانوية ضرورة؛ إعدادًا لهم لاخول الجامعة أو جامعة الحياة. وحسبي أن أؤكد على حقيقة لا يمكن تجاهلها، ونحن على أعتاب العقد الثالث من

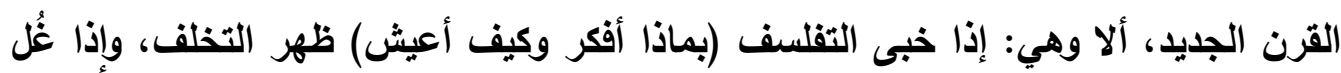
التفكير انطلقت عصابات التكفير (1'). 
وإذا كنا جادين في حماية الأجيال الجديدة من أبنائنا من مخاطر التفكير غير السليم،

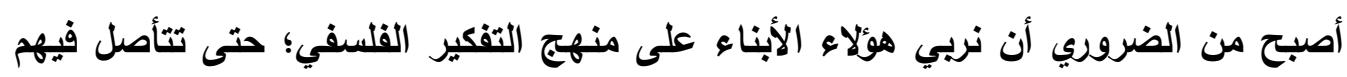

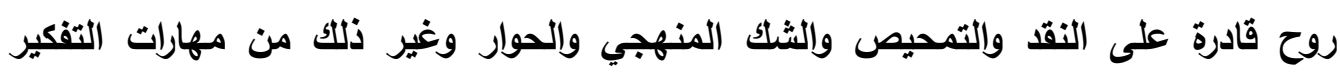

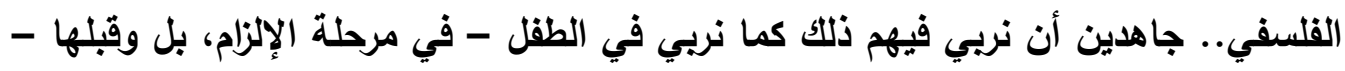

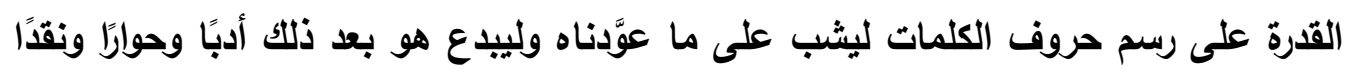

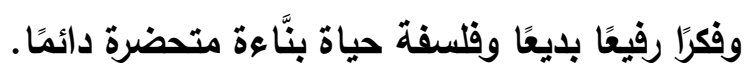

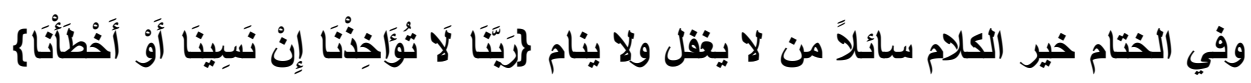

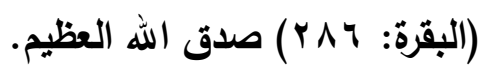




\section{المراجع}

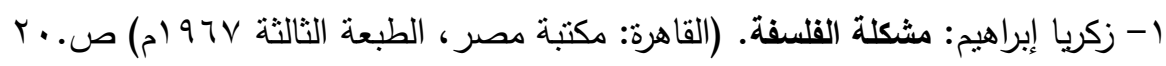
ץ- محمود زقزوق: ما الاي يمكن أن تقدمه القلسفة الإسلامية لبناء الإنسان؟.. جريدة الأهرام (العدد

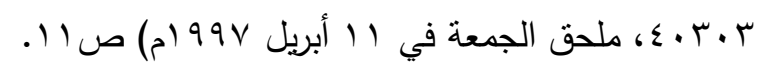

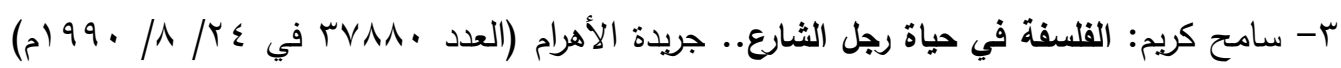
ص.

ع- عبد الغفار مكاوي: مدرسة المحكمة. (القاهرة: دار الكاتب العربي للطباعة والنشر Vד (ام) ص. ه- نقلاً عن: محمد سيد أحمد المسير: المجتمع المثالي في الفكر الفلسفي وموقف الإسلام منه. (رسالة دكتوراه، كلية أصول الدين - جامعة الأزهر (منشورة) - دمشق - بيروت: مؤسسة علوم القرآن.

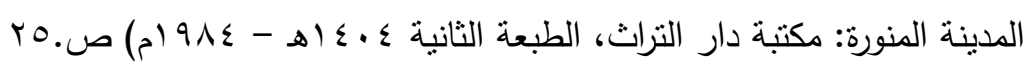
צ- ديكارت: مبادئ القلسفة. نزجمه وقدم له وعلق عليه عثمان أمين. (القاهرة: دار الثقافة للطباعة

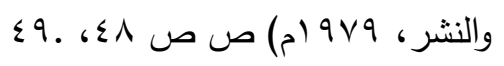
- Vحمد يوسف موسى: القرآن والقلسفة. (دار المعارف بمصر ، 901 (م) ص. ع 7 ᄉ- للمزبد من التفاصيل راجع: - محمد عثمان نجاتي: الحديث النبوي وعلم النفس. (القاهرة: دار الثروق، الطبعة الثانية ساءء اهـ -

$$
\text { ا }
$$

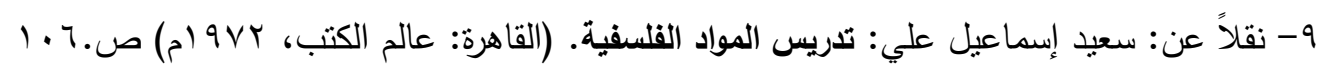
10- Emmet, E. R.: Learning to Philosophize. (New York: Penguin Books, 1984) p. 19.

ا ا- محمد سعيد أحمد زيدان: استراتيجيات حديثة في تدريس علم الاجتماع. (القاهرة: سفير للإعلام

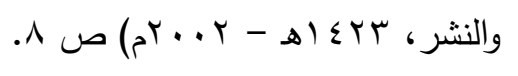

وللمزيد من التفاصيل عن تعليم التفلسف... راجع:

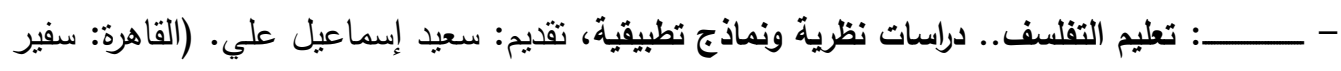

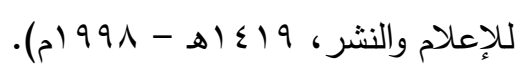

: المثال الثارح مدخل لتعليم التقلسف.. تقديم: حليم فريد نادرس. (القاهرة: سفير للإعلام

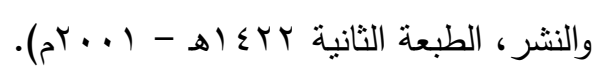


باء الهقال الصحفي مدخل لتعليم التفلسف.. مقالات حليم تادرس الصحفية نموذجًا. (القاهرة: سفير ،

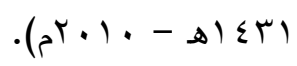

: الفلسفة والحياة اليومية.. المواقف الحياتية مدخل لتدريس المواد القلسفية. (القاهرة: دار مصر

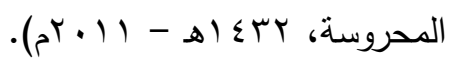

: قراءة نقلية لرسائل الماجستير والدكتوراه في مجال المناهج وطرق تدريس المواد الفلسفية

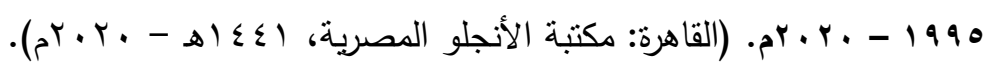

